\title{
Radiotherapy for oligometastatic non-small cell lung cancer: a narrative review
}

\author{
Nora Sundahl, Yolande Lievens \\ Department of Radiation Oncology, Ghent University Hospital \& Ghent University, Ghent, Belgium \\ Contributions: (I) Conception and design: Both authors; (II) Administrative support: None; (III) Provision of study materials or patients: None; \\ (IV) Collection and assembly of data: Both authors; (V) Data analysis and interpretation: Both authors; (VI) Manuscript writing: Both authors; \\ (VII) Final approval of manuscript: Both authors. \\ Correspondence to: Nora Sundahl. Department of Radiation Oncology, Ghent University Hospital, C. Heymanslaan 10, 9000 Ghent, Belgium. \\ Email: nora.sundahl@ugent.be.
}

\begin{abstract}
Preclinical and early clinical evidence suggest that radical radiotherapy of oligometastatic disease in non-small cell lung cancer (NSCLC) patients can impact outcomes with relatively limited toxicity. Whilst data from phase 2 randomized trials suggesting an improved overall survival (OS) with this treatment is promising, it has also illustrated the heterogeneity in this patient population and treatment. Oligometastatic disease in itself comprises a broad spectrum of patients, in terms of tumor load and location, stage of the disease and treatment history. This real-life variety in patient characteristics is often reflected in studies to a certain extent, hinting to the fact that all might benefit from radical radiotherapy to limited metastatic disease, yet leaving the question unanswered as to whom the ideal candidate is. Furthermore, differences between and within studies with regards to treatment modality, timing, radiation technique, and radiation dose are substantial. Also, preclinical and early clinical trials suggest that radiotherapy can work synergistically with checkpoint inhibitors by acting as an in situ cancer vaccine, therefore the combination of these two treatments in oligometastatic patients might entail the largest benefit. Ongoing randomized controlled phase 3 trials and prospective registry trials will further elucidate the true extent of benefit of this local treatment strategy and aid in identifying the ideal patient population and therapy. The current narrative review summarizes the clinical evidence on radiotherapy for oligometastatic NSCLC and highlights the remaining unknowns.
\end{abstract}

Keywords: Oligometastatic; non-small cell lung cancer (NSCLC); radiotherapy

Submitted Sep 16, 2020. Accepted for publication Mar 17, 2021.

doi: $10.21037 /$ tlcr-20-1051

View this article at: http://dx.doi.org/10.21037/tlcr-20-1051

\section{Introduction}

Over the past three decades, it became clear that the oligometastatic disease state-as initially described by Hellman and Weichselbaum-is a separate clinical entity on the spectrum of metastatic disease (1). Retrospective studies indicated that patients with metastatic disease most often first progress in sites of known disease rather than in new sites (2-4), leading to the hypothesis that radical treatment of visible lesions might impact the disease course. In the case of limited metastatic disease, eradication of all disease might abrogate further dissemination and could potentially lead to cure. Progress in both radiation and surgical techniques has allowed the relatively safe radical treatment of metastases. Whilst systemic therapy used to be the only mainstay for patients with spread disease, clinical evidence now corroborates the hypothesis that radical treatment of oligometastatic disease might impact survival (5-11).

Whereas some patients with non-small cell lung cancer (NSCLC) may naturally exhibit a more indolent disease course, with development of oligometastatic rather than polymetastic disease - at diagnosis or as a first presentation 
of disease recurrence-progress in systemic therapies, including targeted treatments and checkpoint inhibitors $(12,13)$, has resulted in a selection of patients showing a significantly prolonged disease course in which treatment can sometimes induce an oligometastatic state in initially polymetastatic patients. As a result, it is plausible that the patient population exhibiting an oligometastatic phenotype has grown over the past decades.

Furthermore, the diagnosis of this oligometastatic presentation is predominantly based on imaging (14). Highly sensitive imaging via positron emission tomographycomputed tomography (PET-CT) has been the standard of care imaging technique in patients with NSCLC for over a decade, which has perhaps led to more frequent diagnoses of the oligometastatic disease state in this tumor type as compared to others where less sensitive imaging techniques were used. This may explain the early adoption of studies on radical treatment of oligometastatic disease in this tumor type $(6,15,16)$.

In other disease types, such as prostate cancer, ablative radiotherapy has been given in an attempt to defer systemic therapy and its related negative impact on quality of life (17). Given the limited toxicity, radical radiotherapy of oligometastatic disease might indeed form an appealing option in frail patients, not amenable to aggressive systemic therapy. This may especially hold true for the NSCLC population, given the etiological association with smoking, with many patients having multiple comorbidities and a limited performance status. Still, given the quickly progressing nature of lung cancer, where even in those who can tolerate systemic agents outcome remains grim (18), radical radiotherapy for oligometastatic disease is typically given as an additional treatment with the aim of consolidating or prolonging the effect of systemic therapy $(5,6,15,16)$. Moreover, data are emerging suggesting that radiotherapy might also be able to synergize and reinforce systemic treatment.

The current article reviews clinical evidence on radiotherapy in oligometastatic NSCLC patients, and digs deeper into the interplay between patient and tumor characteristics, and radiation techniques and doses utilized for this treatment, whilst highlighting the remaining unknowns. Literature was searched via PubMed using the terms 'non-small cell lung cancer', 'oligometastastic', 'radiotherapy', 'radical treatment' and variants thereof, whilst focusing on prospective trials. Additional relevant publications were found by hand searching reference lists. We present the following article in accordance with the Narrative Review reporting checklist (available at http:// dx.doi.org/10.21037/tlcr-20-1051).

\section{Clinical evidence}

Multiple single-arm prospective studies, three randomized phase II trials and the interim results of a phase III randomized controlled trial evaluating local consolidative treatment in patients with oligometastatic NSCLC have been reported (https://cdn.amegroups.cn/static/public/tlcr20-1051-1.pdf).

Gomez et al. $(6,7)$ performed a randomized phase II trial, comparing local consolidative therapy to standard maintenance treatment or observation in NSCLC patients without progression after approximately three months of first line treatment, being chemotherapy or epidermal growth factor receptor (EGFR) inhibitors (in case of EGFR mutation) or anaplastic lymphoma kinase (ALK) inhibitors (in case of ALK rearrangement). Patients with a maximum of three (extra- or intracranial) metastases after three months of systemic treatment were allowed, hence including both genuine and induced oligometastatic disease (19). Regional nodal disease was allowed and collectively counted as one lesion. Consolidative therapy could be either surgery or radiotherapy, whereby stereotactic radiotherapy, hypofractionated radiotherapy and concurrent chemoradiotherapy were options; dose-fractionation regimens were at physician's discretion. The trial was terminated early after randomization of 49 patients, as an interim analysis indicated a $99 \%$ probability of superiority of the experimental arm. A median progression-free survival (PFS) of 14.2 months was noted in the local consolidative therapy arm, versus 4.4 months in the control arm, translating into a significant overall survival (OS) benefit (median of 41.2 months OS in the experimental arm versus 17 months in the control arm). Importantly, this OS benefit was observed even though crossover was permitted, with 9 of 24 patients in the control arm receiving local consolidative therapy upon progression. This suggests that local consolidative therapy is ideally given early in the disease course, without waiting for progression. Yet, even delayed local ablation given at the time of progression partly compensated for the loss in outcome of not giving immediate treatment, be it with the risk of losing a subset of patients to polymetastatic disease or declining performance status. Notably, time to appearance of a new lesion was also longer in the experimental arm, being 14.2 months as compared to 6.0 months. This again suggests that residual macroscopic tumor deposits may be the first focus 
of subsequent metastatic spread, that can be prevented or delayed by local therapy. No grade 4 or 5 adverse events were noted.

A similar phase II trial by Iyengar et al. (5) was conducted in NSCLC patients with up to six extracranial disease sites, including the primary, without progressive disease after induction chemotherapy. Patients with targetable genetic changes or uncontrolled/untreated intracranial disease were not allowed. Randomization was performed between maintenance chemotherapy and stereotactic body radiotherapy (SBRT) or hypofractionated radiotherapy to all visible disease followed by maintenance chemotherapy. Twenty-nine patients were included and randomized. Median PFS was significantly longer in the experimental arm at 9.7 months, as compared to 3.5 months in the maintenance chemotherapy arm. Median OS was not reached in the experimental arm and 17 months in the control arm. No grade 4 or 5 toxicity was reported in the radiotherapy arm.

The phase III SINDAS trial (8) recently reported interim results. This trial compared first-line TKI with or without upfront local radiotherapy in oligometastatic EGFR mutated NSCLC patients. Patients were allowed to have a maximum of five metastatic lesions. SBRT was administered at a dose of 25-40 Gray (Gy) in five fractions to all disease sites. A planned interim analysis after randomization of 133 patients showed a significantly improved PFS from 12.5 to 20.2 months, as well as an improved OS from 17.4 to 25.5 months. No excess toxicity was noted between arms, no grade 5 events were observed. Of interest, so far 631 patients were screened for this trial, illustrating the difficulty of finding the correct patient population. Screening numbers are unfortunately not available for the trials by Gomez et al. and Iyengar et al.

The SABR-COMET trial $(9,10)$ was a randomized phase II basket trial in patients with a maximum of five extraor intracranial metastases and a previously treated and controlled primary tumor of any origin. Randomization occurred 2:1 to either SBRT to all metastases and standard of care systemic treatment, or standard of care systemic treatment alone. Doses for SBRT ranged from 30-60 Gy in 3-8 fractions, or 16-24 Gy in a single fraction for brain or vertebral metastases. Ninety-nine patients were enrolled, of which 66 in the experimental arm. In both arms, $18 \%$ of patients had a primary lung cancer, yet it is unknown how many of these patients had NSCLC. PFS was significantly longer in the experimental arm as compared to the control arm, at 11.6 versus 5.4 months respectively. Median OS was significantly improved to a larger extent than PFS from 28 months in the control arm to 50 months in the experimental arm. Even though baseline characteristics between both arms were not balanced with more prostate cancer patients in the experimental arm, a post hoc sensitivity analysis excluding these patients continues to show a significant survival improvement due to the addition of SBRT. In contrast to the findings of Gomez et al., no difference was noted between arms for the time to new metastasis. In this trial, consolidative radiotherapy unfortunately came at a cost: SBRT treatment was associated with a $20 \%$ increase in grade 2 or higher toxicity, while three patients experienced grade 5 toxicity, with their death possibly, probably or definitely related to treatment.

The majority of trials did not observe excess toxicity in the radiotherapy arm, which is corroborated by single arm prospective trials reporting grade 3 adverse events generally in less than $10 \%$ of patients, and grade 4 adverse events only very rarely $(3,5,8,15,20-25)$. In contrast, higher toxicity was noted in the SABR-COMET trial, the trial by Gomez et al. and the trial by De Ruysscher et al. $(6,7,9,10,16)$ Most strikingly, the SABR-COMET trial $(9,10)$ reported a $20 \%$ increase in grade 2 or higher toxicity, observed in $9 \%$ of patients in the control arm versus $29 \%$ of patients in the SBRT arm. Grade 3 toxicity was limited, with dyspnea and pain being observed in the SBRT arm in only 1 and 3 patients respectively; in the control arm one patient with grade 3 fatigue was the only grade 3 adverse event. Sadly, three deaths occurred in the SBRT arm, i.e., due to a radiation pneumonitis after SBRT to a central lung metastasis, a pulmonary abscess at the location of previous SBRT, and a subdural hemorrhage occurring after reparative surgery for an SBRT-related perforated gastric ulcer. The earliest single-arm prospective trial by De Ruysscher et al. (16) investigated upfront radical treatment, be it (a combination of) surgery, radiotherapy or radiochemotherapy, of all visible disease-including the primary - in 39 newly diagnosed oligometastatic NSCLC patients. No grade 4 or 5 toxicity was observed, yet $15 \%$ of patients experienced grade 3 oesophagitis and one patient had a grade 3 cough. In the trial by Gomez et al., 5 out of 25 patients in the experimental arm had grade 3 adverse events, as compared to 2 patients in the control arm $(6,7)$. These findings suggest that whilst radiotherapy to visible disease in oligometastatic patients is tolerated well in the majority of patients, a small risk for severe, potentially 
lethal, toxicity exists.

\section{Radiation techniques and the search for the ideal dose}

Radiation treatments in the studies varied from SBRT, to hypofractionated radiotherapy and conventional radio(chemo)therapy, where the latter may have been delivered with $3 \mathrm{D}$-conformal radiotherapy as well as using intensity modulations. Given this discrepancy and lack of randomization between techniques, it is impossible to ascertain whether there is a preferred-more effectiveradiotherapy approach in this setting (3,5-10,15,16,20-25).

In parallel to the techniques used and the association to chemotherapy—or not—-the administered doses varied substantially between and within studies (3,5-10,15,16,20-25), ranging from a biologically effective dose (BED) of 39 Gy (for $30 \mathrm{~Gy}$ in ten fractions) to $151 \mathrm{~Gy}$ (for $54 \mathrm{~Gy}$ in three fractions) (for an $\alpha / \beta=10$ ). Local control of lesions treated with radiotherapy in these trials varied between $63 \%$ and $100 \%$ and was better than in the control arms $(5-7,9,10)$. Even though multiple retrospective and prospective series in metastases from various primary tumors indicate better local control with higher biologically effective radiation doses $(6,22,26-37)$, further prospective data regarding the ideal dose is awaited. Higher doses resulting in better local control was not clearly observed in the majority of prospective trials including lung cancer patients, nor was it evident when comparing between trials $(3,5-10,15,16,20-25)$. A previous retrospective analysis of SBRT for lung metastases has suggested the dose-response plateaus at 160 Gy BED [planning target volume (PTV) maximum dose], yielding a tumor control probability of $90 \%$. If administered in three fractions, this corresponds to the PTV encompassing doses of 11.2-16.8 Gy (60-90\% of the maximum dose) (38). The safety of such high SBRT doses in, for instance, lung oligometastases has been extrapolated from stage I NSCLC and was confirmed in three dose-escalation studies in the oligometastatic setting $(20,22,25,38)$. As suggested by Guckenberger et al. (38), it is however uncertain whether the maximum tolerated dose in the setting of oligometastatic disease is preferable, as the evidence is based on phase I trials where patients on average had 1-2 lung metastases. Yet in everyday clinic, many patients have more than one or two metastases to be treated, and are likely to progress at a certain point, perhaps needing further local radiotherapy to other lesions. Furthermore, as is evidenced by the SABR-COMET trial, there is a risk, albeit small, for important—even lethal—toxicity $(9,10)$, and previous reports have shown that the higher the dose, the higher the risk of toxicity (39).

Even though tumor control probability at a certain radiation dose is likely to be similar regardless of the location of the metastasis, similar radiation doses $(40,41)$ do not entail the same toxicity risk in different organs or for different lesion sizes. For instance, a safe dose for a small peripheral lung metastasis (e.g., 60 Gy in three fractions), would be too toxic and unacceptable for a very large peripheral lung metastasis, or a gastro-intestinal or central lung metastasis. In the latter situation, it is necessary to limit the radiation dose to ensure a safe treatment, which unfortunately will reduce the probability of tumor control, similar to what has been observed in risk-adapted SBRT for early-stage NSCLC (42). The SABR-COMET trial also showed that even when dose prescriptions are altered and reduced to meet normal tissue safety constraints, toxicity cannot be ruled out, illustrating the difficulty of risk prediction $(9,10)$.

The ideal radiation dose should therefore balance both the benefit of tumor control probability and the risk of toxicity in light of the lesion location, the lesion size, and potential multiple radiation courses, with safety of SBRT being the absolute priority (32).

\section{Outcome per location: an intricate interplay of dose, volume and primary tumor}

The location and size of the oligometastasis will therefore affect the deliverable radiation dose, which in turn influences the tumor control probability. This interplay can explain why different local control rates are observed based on the organ of the metastasis. Furthermore, the evidence concerning these local control rates tends to come from multiple different tumor types with varying radiosensitivity, in patients who may or may not have been receiving systemic treatment. All of these factors will alter the tumor control probability, adding other layers of complexity.

The SABR-COMET trial noted different local control rates depending on the organ of the metastasis, with $100 \%$ for adrenal metastases, $72 \%$ for bone metastases, $51 \%$ for lung metastases and $50 \%$ for liver metastases $(9,10)$. For adrenal metastases, this high local control rate is however not concordant with previous reports, showing 1- and 2-year local control rates after radiotherapy for adrenal metastases of $55-73 \%(43-45)$ and $27-46.6 \%$ (44-47), respectively. This is contrary to bone metastases, where in general high local control rates after radiotherapy have been reported, 
ranging between $80-96 \%$ and $73-93 \%$ at 1 - and 2 -year, respectively (48-57).

Nonetheless, a handful of retrospective series on metastatic NSCLC patients with bone metastases unfortunately suggest poor outcomes for these patients with a 2 -year survival of only $13 \%$ (58-61). The discrepancy between these good local control rates and poor outcomes is presumably due to the limited amount of data available for this specific population, making treatment recommendations difficult (58-61).

Rusthoven et al. reported on a phase I/II dose escalation trial in patients with pulmonary metastases. SBRT dose was escalated from 48 to 60 Gy in three fractions in 38 patients (of whom five with a primary lung tumor). Local control rates were excellent at $100 \%$ and $96 \%$ at 1 - and 2 -year follow-up respectively (20). Similar high local control rates in the order of magnitude of $90 \%$ at 1 year and $80 \%$ at 2 years have been reported in retrospective studies, again including a multitude of primary tumors (39,62-64). De Rose et al. reported on 60 NSCLC patients with 90 lung metastases treated with SBRT (64). SBRT dose was $60 \mathrm{~Gy}$ in three to eight fractions for lesions up to $2 \mathrm{~cm}$, and 48 Gy in four fractions for lesions between $2-5 \mathrm{~cm}$. Using this dose-adapted scheme to mitigate toxicity, a 2-year local control rate of $88.9 \%$ was observed. Of interest, these high local control rates are in the same order of magnitude as what has been reported in early-stage NSCLC treated with SBRT (65). In a retrospective multi-centre series of 399 patients with primary NSCLC and 397 patients with pulmonary metastases from various primary origins, similar tumor control probabilities were found in both groups (38).

As for liver metastases, a phase I/II trial assessed the safety and local control of SBRT to one to three hepatic metastases. Dose was escalated from 36 to $60 \mathrm{~Gy}$ in three fractions, leading to a local control rate of $95 \%$ and $92 \%$ at 1 and 2 years follow-up respectively (21). Local control was $100 \%$ in lesions below $3 \mathrm{~cm}$, but dropped to $77 \%$ for lesions beyond $3 \mathrm{~cm}$. A large retrospective analysis of 474 patients who received SBRT to liver metastases, including 29 NSCLC patients, showed a 1-year local control rate of $76.1 \%$ for the whole group and $88 \%$ for the NSCLC patients (26). This large, real-life multicenter cohort illustrates how differences in dose prescriptions, concomitant systemic therapy and primary tumor histology can affect the outcome. The good local control rate in this study is corroborated by a meta-analysis of retrospective studies comparing SBRT to radiofrequency ablation (RFA) for liver metastases of various origins, showing a 2-year local control rate of $83.6 \%$ for SBRT (66).

Patients with intracranial oligometastases are often studied and reported separately from those with extracranial oligometastases, even though consensus converges towards the inclusion of these patients in future studies on oligometastatic disease (32). Several retrospective analyses have reported on patients with NSCLC and oligometastastic synchronous brain metastases treated with radiotherapy (67-71). Yang et al. focused on patients with thoracic stage I or II NSCLC and one to three synchronous brain metastases without extracranial metastases. All patients underwent Gamma Knife radiosurgery for their intracranial disease to a dose of 15 to $25 \mathrm{~Gy}$, with or without thoracic surgery to the primary. Only two of 31 patients had local intracranial failure, resulting in a $97.1 \%$ and $93.5 \% 1$ - and 2-year local control rate for metastatic brain tumors (70). Hu et al. (69) reported on patients with a single brain metastasis receiving stereotactic radiosurgery $(n=31)$ or neurosurgical resection $(n=53)$. Local control rates at 1 and 2 years were both $61 \%$ for the radiosurgery group, compared to $97 \%$ and $88 \%$ for the neurosurgical group, respectively. However, due to the retrospective nature of this study, this difference in local control between treatment modality might be due to patient selection. Unfortunately, no randomized data exists comparing radiosurgery to neurosurgery for limited intracranial metastases.

Similarly, no head-to-head comparison exists between radiotherapy and surgery or RFA for extracranial metastatic disease (47). A recent meta-analysis of retrospective studies suggests that SBRT may lead to improved 2-year local control rates as compared to RFA for liver metastases, at $83.6 \%$ and $60 \%$ respectively (66). This difference in outcome seems to especially hold for lesions larger than $2 \mathrm{~cm}$ (72). In retrospective studies, surgery tends to either have similar or better outcomes than radiotherapy $(62,66,73)$. Yet, when comparing different treatment modalities using retrospective data, the results are inherently biased. Even if baseline patient characteristics are matched, there remain unknown variables which can impact on prognosis regardless of treatment. Patients fit for surgery tend to have a better prognosis from the start, with a better performance status, less comorbidities and smaller metastases. This is supported by a study by Soni et al. (74) which compared results of observational studies in the oncological field, to results of subsequent randomized controlled trials seeking to answer the same question. This showed that observational studies are more likely to favor outcomes after surgery than after radiotherapy; a pattern not observed in randomized controlled trials. Hence, in 
the absence of randomized controlled trials comparing SBRT to other loco-regional treatment strategies, no firm conclusions can be drawn.

\section{How to select the right patient that will benefit from radiotherapy?}

Whom is regarded as an oligometastatic patient and which treatments are offered tends to differ from physician to physician and has shifted over time, with a tendency to reserve radical local therapy such as SBRT for patients not progressing after systemic therapy (75). Furthermore, as compared to the theoretical definition of oligometastatic disease based on number of lesions, in the real-life setting, physicians tend to be more restrictive as to who they label as oligometastatic (75). Multiple studies have evaluated and suggested clinical prognostic markers to guide patient selection (19,76-79), however, these are all based on retrospective data, and have not yet been prospectively validated. It also remains uncertain whether these prognostic factors merely allow selection of patient groups with a better prognosis per se, or whether they can truly predict who will benefit from radical treatment of their oligometastases. Aside from the fact that the cutoff of oligometastatic disease is generally arbitrarily set to a maximum of three or five metastases, this clinical and radiological entity that is referred to as 'oligometastatic disease' comprises a broad and variable spectrum of disease states, with presumably substantially different underlying tumor biology (19). This variability is also reflected in the prospective and randomized trials evaluating the benefit of local consolidative therapy in NSCLC patients with limited metastatic burden, which often included patients from different parts of the oligometastatic spectrum, based on divergent inclusion criteria (https://cdn.amegroups.cn/ static/public/tlcr-20-1051-1.pdf) (3,5-10,15,16,20-25).

The maximum number of metastases, the activity of the lesions (i.e., new, stable or progressing), the setting (i.e., synchronous, metachronous, or induced oligometastasis, or oligorecurrent, oligopersistent or oligoprogressive), all or not in the context of systemic treatment and whether or not regional nodal metastases were included in the number of lesions; all these factors showed high variability (3,5-10,15,16,20-25). Gomez et al. $(6,7)$ allowed patients with up to three synchronous or metachronous metastatic lesions and counted the lesions after first-line systemic treatment, hence allowing patients with prior polymetastatic disease. Regional nodal disease was collectively counted as one lesion. The trial by Petty et al. (3) allowed patients with up to five synchronous or metachronous metastatic lesions with a maximum of three lesions in any extracranial organ. Regional nodal disease was allowed and was not counted towards this number. De Ruysscher et al. (16) only allowed patients with synchronous oligometastatic disease with a maximum of five metastases, yet strikingly the eventual patient population was more restrictive, consisting of $87.2 \%$ of patients with a single metastasis, $10.3 \%$ with two metastases, $2.6 \%$ with three metastases and none with more. Regional nodal disease was likely not counted as metastatic disease. The randomized trial by Iyengar et al. (5) included patients with a maximum of six synchronous extracranial metastases, including the primary but not involving the gastro-intestinal tract or the skin. The single arm trial by the same group (15) included patients with the same number and sites of metastases, yet progressive after at least one line of chemotherapy. It is uncertain whether induced oligometastatic disease was allowed in this trial. Important to note, even though the extent and number of nodal metastases are often disregarded in trials, in other words, accounted for in the loco-regional disease, their presence might significantly impact the prognosis (80). A recent pan-European consensus report recommended that mediastinal lymph nodes should not be considered as a metastatic site in oligometastatic NSCLC, yet should be regarded as regional disease. They do however acknowledge that the extent of nodal disease impacts radical treatment options, as was previously also suggested on the basis of a meta-analysis $(79,81)$. Further prospective evidence on this topic remains necessary. Furthermore, not only the extent of local disease is of importance, but also whether or not the disease is controlled. There is a discrepancy between trials whether or not patients with active and progressing local disease were allowed and if so, how this was treated (3,5-10,15,16,20-25). Also, not all trials mandated the use of PET-CT imaging to determine the number of metastases, while using less sensitive imaging might lead to different oligometastatic patient populations. For example, a patient with three metastases on CT imaging might have additional small PET-avid lesions, occult on CT, and hence only be regarded as oligometastatic when using CT imaging, not when using PET-CT. By using less sensitive imaging patients will be down-staged and the resultant oligometastatic population will inherently have a worse prognosis than the oligometastatic population based on PET-CT (82).

From the above it becomes evident that the large heterogeneity in clinical practice $(75,83)$ is reflected in 
prospective radiotherapy research on oligometastatic disease where the included patients are heterogeneous between and within trials $(3,5-10,15,16,20-25)$. This hints to the fact that a variety of patients might benefit from local radiotherapy to visible disease, yet leaves us in the dark as to whom the ideal candidates are. Targeted biomarkers aiding in patient selection for this treatment would be useful, yet are unfortunately still awaited.

\section{Future perspectives in this rapidly evolving field}

Results from randomized phase 2 trials suggest that radical treatment of oligometastases in NSCLC patients can prolong OS. Yet, multiple phase 3 trials, such as the SABRCOMET-3, SARON and OMEGA trial, are currently ongoing and awaited to confirm this benefit.

As previously stated, current unresolved issues include the ideal treatment modality. Radiotherapy, surgery and potentially RFA are likely to be options for this radical treatment, as prospective trials have allowed different treatment modalities without indication of a clear benefit of any treatment modality over another. Further evidence to better define the choice for one or the other treatment modality would be optimal, but seeing the large variation in clinical presentation, in a population with often important co-morbidities, the potential benefit and harms of a treatment should always be weighed on a patient by patient basis. This can best be obtained through multidisciplinary team discussion, in the context of a comprehensive cancer center $(75,84)$.

The ideal timing of radiotherapy both in the disease course and relative to systemic treatment is yet to be determined. The trial by Gomez et al., indicating a survival benefit for local consolidative treatment, allowed crossover of patients in the control arm to local consolidative treatment upon progression, which nine of 24 patients in this arm received $(6,7)$. Almost a third of patients in the control arm however progressed into polymetastatic disease, suggesting that radical treatment of oligometastases early in the disease course is key in order not to miss the window of opportunity. Whether or not systemic treatment should be administered sequentially, concomitantly or be withheld when considering radical treatment of visible disease, is unknown. Preclinical and early clinical data suggest that radiotherapy might work in synergy with immunotherapy (85-89). The hypothesis goes that whilst the brake on the immune system is released by checkpoint inhibitors, local radiotherapy instigates the release of tumor-specific antigen and ignites a pro-inflammatory tumor micro-environment, aiding recognition of the malignant cells locally and distant to the irradiated lesion. The latter is coined the 'abscopal' effect (89). Preclinical data suggests that hypofractionated radiotherapy, e.g., $24 \mathrm{~Gy}$ in three fractions would be ideal in triggering this effect (90). The accumulating evidence on immunotherapy in NSCLC has recently led to a change in practice in advanced and in locally-advanced disease $(91,92)$. While in the latter the addition of immunotherapy to concurrent chemoradiotherapy has already become the new standard, in oligometastatic disease, radiotherapy to all or part of the macroscopic lesions in combination with immunotherapy might similarly herald advantages in long-term survival through local synergistic effects in the irradiated lesions and a systemic abscopal response in microscopic disease (93).

One of the main questions remains who the ideal candidates are for radical (radiation) treatment of oligometastatic disease. The performed trials often included a highly selected patient population, as illustrated by the SINDAS trial where only about 1 out of 5 screened patients were enrolled in the study so far (8). It is therefore uncertain how results of these trials can be extrapolated to the patients we encounter in the realworld setting. In addition, while the definition of what truly represents oligometastatic disease remains under scrutiny, the different trials have used these definitions in a variable manner, further adding to the difficulty of selecting the right patients for radical treatment of metastases (94). Hence, more evidence, ideally from randomized controlled trials, is still awaited to further support practice. It can nevertheless be anticipated that the broad spectrum of oligometastatic disease, in lung cancer and other cancers, will be difficult to capture solely by clinical trials. Moreover, the available results have already fueled the uptake of SABR for oligometastatic disease in daily practice (95). In order to capture this momentum and learn from the treatments already being clinically delivered, ESTRO and EORTC have jointly initiated OligoCare under the E2-RADIatE platform (EORTC-ESTRO RADiation InfrAstrucTure for Europe, NCT03818503). This international prospective registry trial is aimed at identifying patient, tumor and treatment characteristics that impact OS of patients with oligometastatic NSCLC, colorectal, prostate or breast cancer, treated with radical radiotherapy to all visible disease. As inclusion criteria are purposely broad, this study will hopefully aid in describing patterns-of-care, identifying relevant prognostic and predictive factors, and defining accessibility and cost of radiotherapy in the current clinical setting (19). In addition, by capturing the data following 
the EORTC oligometastases characterization system (19), it will hopefully broaden our understanding of the impact of radiotherapy in the distinct oligometastatic disease states and generate hypotheses to be further addressed in formal, randomized controlled trials.

\section{Conclusions}

Current clinical evidence from randomized phase 2 trials suggests that radical radiotherapy of oligometastatic disease in NSCLC patients can prolong survival, yet further confirmatory data from randomized controlled phase 3 trials remains awaited. The oligometastatic patient population and the treatments used in trials prove to be heterogeneous, leaving many questions regarding the ideal candidate, treatment modality, timing and radiation dose unanswered. Ongoing and future trials will likely lift the remaining corners of the veil in the upcoming years.

\section{Acknowledgments}

Funding: None.

\section{Footnote}

Provenance and Peer Review: This article was commissioned by the Guest Editors (Maurizio Infante \& Thierry Berghmans) for the series "Oligometastatic NSCLC: definition and treatment opportunities" published in Translational Lung Cancer Research. The article has undergone external peer review.

Reporting Checklist: The authors have completed the Narrative Review reporting checklist. Available at http:// dx.doi.org/10.21037/tlcr-20-1051

Conflicts of Interest: Both authors have completed the ICMJE uniform disclosure form (available at http://dx.doi. org/10.21037/tlcr-20-1051). The series "Oligometastatic NSCLC: definition and treatment opportunities" was commissioned by the editorial office without any funding or sponsorship. NS reports travel support from Bayer, MSD, Bristol-Myers Squibb, and Astellas. YL reports expert positions for AstraZeneca and RaySearch, outside the current work. The authors have no other conflicts of interest to declare.

Ethical Statement: The authors are accountable for all aspects of the work in ensuring that questions related to the accuracy or integrity of any part of the work are appropriately investigated and resolved.

Open Access Statement: This is an Open Access article distributed in accordance with the Creative Commons Attribution-NonCommercial-NoDerivs 4.0 International License (CC BY-NC-ND 4.0), which permits the noncommercial replication and distribution of the article with the strict proviso that no changes or edits are made and the original work is properly cited (including links to both the formal publication through the relevant DOI and the license). See: https://creativecommons.org/licenses/by-nc-nd/4.0/.

\section{References}

1. Hellman S, Weichselbaum RR. Oligometastases. J Clin Oncol 1995;13:8-10.

2. Rusthoven KE, Hammerman SF, Kavanagh BD, et al. Is there a role for consolidative stereotactic body radiation therapy following first-line systemic therapy for metastatic lung cancer? A patterns-of-failure analysis. Acta Oncol 2009;48:578-83.

3. Petty WJ, Urbanic JJ, Ahmed T, et al. Long-Term Outcomes of a Phase 2 Trial of Chemotherapy With Consolidative Radiation Therapy for Oligometastatic Non-Small Cell Lung Cancer. Int J Radiat Oncol Biol Phys 2018;102:527-35.

4. Weichselbaum RR, Hellman S. Oligometastases revisited. Nat Rev Clin Oncol 2011;8:378-82.

5. Iyengar $\mathrm{P}$, Wardak $Z$, Gerber DE, et al. Consolidative Radiotherapy for Limited Metastatic Non-Small-Cell Lung Cancer: A Phase 2 Randomized Clinical Trial. JAMA Oncol 2018;4:e173501.

6. Gomez DR, Blumenschein GR Jr, Lee JJ, et al. Local consolidative therapy versus maintenance therapy or observation for patients with oligometastatic non-smallcell lung cancer without progression after first-line systemic therapy: a multicentre, randomised, controlled, phase 2 study. Lancet Oncol 2016;17:1672-82.

7. Gomez DR, Tang C, Zhang J, et al. Local Consolidative Therapy Vs. Maintenance Therapy or Observation for Patients With Oligometastatic Non-Small-Cell Lung Cancer: Long-Term Results of a Multi-Institutional, Phase II, Randomized Study. J Clin Oncol 2019;37:1558-65.

8. Wang X, Zeng M. First-line tyrosine kinase inhibitor with or without aggressive upfront local radiation therapy in patients with EGFRm oligometastatic non-small cell lung 
cancer: Interim results of a randomized phase III, openlabel clinical trial (SINDAS) (NCT02893332). J Clin Oncol 2020;38:9508.

9. Palma DA, Olson R, Harrow S, et al. Stereotactic ablative radiotherapy versus standard of care palliative treatment in patients with oligometastatic cancers (SABRCOMET): a randomised, phase 2, open-label trial. Lancet 2019;393:2051-8.

10. Palma DA, Olson R, Harrow S, et al. Stereotactic Ablative Radiotherapy for the Comprehensive Treatment of Oligometastatic Cancers: Long-Term Results of the SABR-COMET Phase II Randomized Trial. J Clin Oncol 2020;38:2830-8.

11. Ashworth A, Rodrigues G, Boldt G, et al. Is there an oligometastatic state in non-small cell lung cancer? A systematic review of the literature. Lung Cancer 2013;82:197-203.

12. Garon EB, Rizvi NA, Hui R, et al. Pembrolizumab for the treatment of non-small-cell lung cancer. N Engl J Med 2015;372:2018-28.

13. Zhou C, Wu YL, Chen G, et al. Erlotinib versus chemotherapy as first-line treatment for patients with advanced EGFR mutation-positive non-small-cell lung cancer (OPTIMAL, CTONG-0802): a multicentre, open-label, randomised, phase 3 study. Lancet Oncol 2011;12:735-42.

14. Lecouvet FE, Oprea-Lager DE, Liu Y, et al. Use of modern imaging methods to facilitate trials of metastasisdirected therapy for oligometastatic disease in prostate cancer: a consensus recommendation from the EORTC Imaging Group. Lancet Oncol 2018;19:e534-45.

15. Iyengar P, Kavanagh BD, Wardak Z, et al. Phase II trial of stereotactic body radiation therapy combined with erlotinib for patients with limited but progressive metastatic non-small-cell lung cancer. J Clin Oncol 2014;32:3824-30.

16. De Ruysscher D, Wanders R, van Baardwijk A, et al. Radical treatment of non-small-cell lung cancer patients with synchronous oligometastases: long-term results of a prospective phase II trial (Nct01282450). J Thorac Oncol 2012;7:1547-55

17. Ost P, Reynders D, Decaestecker K, et al. Surveillance or Metastasis-Directed Therapy for Oligometastatic Prostate Cancer Recurrence: A Prospective, Randomized, Multicenter Phase II Trial. J Clin Oncol 2018;36:446-53.

18. Herbst RS, Baas P, Kim DW, et al. Pembrolizumab versus docetaxel for previously treated, PD-L1-positive, advanced non-small-cell lung cancer (KEYNOTE-010): a randomised controlled trial. Lancet 2016;387:1540-50.

19. Guckenberger M, Lievens Y, Bouma AB, et al. Characterisation and classification of oligometastatic disease: a European Society for Radiotherapy and Oncology and European Organisation for Research and Treatment of Cancer consensus recommendation. Lancet Oncol 2020;21:e18-28.

20. Rusthoven KE, Kavanagh BD, Burri SH, et al. Multiinstitutional phase I/II trial of stereotactic body radiation therapy for lung metastases. J Clin Oncol 2009;27:1579-84.

21. Rusthoven KE, Kavanagh BD, Cardenes H, et al. Multiinstitutional phase I/II trial of stereotactic body radiation therapy for liver metastases. J Clin Oncol 2009;27:1572-8.

22. Salama JK, Hasselle MD, Chmura SJ, et al. Stereotactic body radiotherapy for multisite extracranial oligometastases: final report of a dose escalation trial in patients with 1 to 5 sites of metastatic disease. Cancer 2012;118:2962-70.

23. Collen C, Christian N, Schallier D, et al. Phase II study of stereotactic body radiotherapy to primary tumor and metastatic locations in oligometastatic nonsmall-cell lung cancer patients. Ann Oncol 2014;25:1954-9.

24. Bauml JM, Mick R, Ciunci C, et al. Pembrolizumab After Completion of Locally Ablative Therapy for Oligometastatic Non-Small Cell Lung Cancer: A Phase 2 Trial. JAMA Oncol 2019;5:1283-90.

25. Milano MT, Katz AW, Muhs AG, et al. A prospective pilot study of curative-intent stereotactic body radiation therapy in patients with 5 or fewer oligometastatic lesions. Cancer 2008;112:650-8.

26. Andratschke N, Alheid H, Allgäuer M, et al. The SBRT database initiative of the German Society for Radiation Oncology (DEGRO): patterns of care and outcome analysis of stereotactic body radiotherapy (SBRT) for liver oligometastases in 474 patients with 623 metastases. BMC Cancer 2018;18:283.

27. Guckenberger M, Klement RJ, Allgäuer M, et al. Local tumor control probability modeling of primary and secondary lung tumors in stereotactic body radiotherapy. Radiother Oncol 2016;118:485-91.

28. Guckenberger M, Wulf J, Mueller G, et al. Doseresponse relationship for image-guided stereotactic body radiotherapy of pulmonary tumors: relevance of 4D dose calculation. Int J Radiat Oncol Biol Phys 2009;74:47-54.

29. McCammon R, Schefter TE, Gaspar LE, et al. Observation of a dose-control relationship for lung and liver tumors after stereotactic body radiation therapy. Int J 
Radiat Oncol Biol Phys 2009;73:112-8.

30. Stinauer MA, Kavanagh BD, Schefter TE, et al. Stereotactic body radiation therapy for melanoma and renal cell carcinoma: impact of single fraction equivalent dose on local control. Radiat Oncol 2011;6:34.

31. Stragliotto CL, Karlsson K, Lax I, et al. A retrospective study of SBRT of metastases in patients with primary sarcoma. Med Oncol 2012;29:3431-9.

32. Lievens Y, Guckenberger M, Gomez D, et al. Defining oligometastatic disease from a radiation oncology perspective: An ESTRO-ASTRO consensus document. Radiother Oncol 2020;148:157-66.

33. Bowden P, See AW, Frydenberg M, et al. Fractionated stereotactic body radiotherapy for up to five prostate cancer oligometastases: Interim outcomes of a prospective clinical trial. Int J Cancer 2020;146:161-8.

34. Lépinoy A, Silva YE, Martin E, et al. Salvage extended field or involved field nodal irradiation in $18 \mathrm{~F}$-fluorocholine PET/CT oligorecurrent nodal failures from prostate cancer. Eur J Nucl Med Mol Imaging 2019;46:40-8.

35. Santini D, Ratta R, Pantano F, et al. Outcome of oligoprogressing metastatic renal cell carcinoma patients treated with locoregional therapy: a multicenter retrospective analysis. Oncotarget 2017;8:100708-16.

36. Frelinghuysen M, Schillemans W, Hol L, et al. Acute toxicity of the bowel after stereotactic robotic radiotherapy for abdominopelvic oligometastases. Acta Oncol 2018;57:480-4.

37. Klement RJ, Hoerner-Rieber J, Adebahr S, et al. Stereotactic body radiotherapy (SBRT) for multiple pulmonary oligometastases: Analysis of number and timing of repeat SBRT as impact factors on treatment safety and efficacy. Radiother Oncol 2018;127:246-52.

38. Guckenberger M, Klement RJ, Allgäuer M, et al. Local tumor control probability modeling of primary and secondary lung tumors in stereotactic body radiotherapy. Radiother Oncol 2016;118:485-91.

39. Rieber J, Streblow J, Uhlmann L, et al. Stereotactic body radiotherapy (SBRT) for medically inoperable lung metastases-A pooled analysis of the German working group "stereotactic radiotherapy". Lung Cancer 2016;97:51-8.

40. Oh D, Ahn YC, Seo JM, et al. Potentially curative stereotactic body radiation therapy (SBRT) for single or oligometastasis to the lung. Acta Oncol 2012;51:596-602.

41. Sharma A, Duijm M, Oomen-de Hoop E, et al. Survival and prognostic factors of pulmonary oligometastases treated with stereotactic body radiotherapy. Acta Oncol 2019;58:74-80.
42. Lagerwaard FJ, Haasbeek CJ, Smit EF, et al. Outcomes of risk-adapted fractionated stereotactic radiotherapy for stage I non-small-cell lung cancer. Int J Radiat Oncol Biol Phys 2008;70:685-92.

43. Rudra S, Malik R, Ranck MC, et al. Stereotactic body radiation therapy for curative treatment of adrenal metastases. Technol Cancer Res Treat 2013;12:217-24.

44. Chawla S, Chen Y, Katz AW, et al. Stereotactic body radiotherapy for treatment of adrenal metastases. Int J Radiat Oncol Biol Phys 2009;75:71-5.

45. Scorsetti M, Alongi F, Filippi AR, et al. Long-term local control achieved after hypofractionated stereotactic body radiotherapy for adrenal gland metastases: a retrospective analysis of 34 patients. Acta Oncol 2012;51:618-23.

46. Celik E, Semrau R, Baues C, et al. Robot-assisted Extracranial Stereotactic Radiotherapy of Adrenal Metastases in Oligometastatic Non-small Cell Lung Cancer. Anticancer Res 2017;37:5285-91.

47. Wujanto C, Vellayappan B, Siva S, et al. Stereotactic Body Radiotherapy for Oligometastatic Disease in Non-small Cell Lung Cancer. Front Oncol 2019;9:1219.

48. Zeng KL, Tseng CL, Soliman H, et al. Stereotactic Body Radiotherapy (SBRT) for Oligometastatic Spine Metastases: An Overview. Front Oncol 2019;9:337.

49. Anand AK, Venkadamanickam G, Punnakal AU, et al. Hypofractionated stereotactic body radiotherapy in spinal metastasis - with or without epidural extension. Clin Oncol (R Coll Radiol) 2015;27:345-52.

50. Bate BG, Khan NR, Kimball BY, et al. Stereotactic radiosurgery for spinal metastases with or without separation surgery. J Neurosurg Spine 2015;22:409-15.

51. Guckenberger M, Mantel F, Gerszten PC, et al. Safety and efficacy of stereotactic body radiotherapy as primary treatment for vertebral metastases: a multi-institutional analysis. Radiat Oncol 2014;9:226.

52. Chang UK, Cho WI, Kim MS, et al. Local tumor control after retreatment of spinal metastasis using stereotactic body radiotherapy; comparison with initial treatment group. Acta Oncol 2012;51:589-95.

53. Gill B, Oermann E, Ju A, et al. Fiducial-free CyberKnife stereotactic body radiation therapy (SBRT) for single vertebral body metastases: acceptable local control and normal tissue tolerance with 5 fraction approach. Front Oncol 2012;2:39.

54. Yamada Y, Bilsky MH, Lovelock DM, et al. Highdose, single-fraction image-guided intensity-modulated radiotherapy for metastatic spinal lesions. Int J Radiat Oncol Biol Phys 2008;71:484-90. 
55. Tseng CL, Soliman H, Myrehaug S, et al. Imaging-Based Outcomes for 24 Gy in 2 Daily Fractions for Patients with de Novo Spinal Metastases Treated With Spine Stereotactic Body Radiation Therapy (SBRT). Int J Radiat Oncol Biol Phys 2018;102:499-507.

56. Bishop AJ, Tao R, Rebueno NC, et al. Outcomes for Spine Stereotactic Body Radiation Therapy and an Analysis of Predictors of Local Recurrence. Int J Radiat Oncol Biol Phys 2015;92:1016-26.

57. Wang XS, Rhines LD, Shiu AS, et al. Stereotactic body radiation therapy for management of spinal metastases in patients without spinal cord compression: a phase 1-2 trial. Lancet Oncol 2012;13:395-402.

58. Takahashi Y, Adachi H, Mizukami Y, et al. Patient outcomes post-pulmonary resection for synchronous bone-metastatic non-small cell lung cancer. J Thorac Dis 2019;11:3836-45.

59. Zhang L, Gong Z. Clinical Characteristics and Prognostic Factors in Bone Metastases from Lung Cancer. Med Sci Monit 2017;23:4087-94.

60. Griffioen GH, Toguri D, Dahele M, et al. Radical treatment of synchronous oligometastatic non-small cell lung carcinoma (NSCLC): patient outcomes and prognostic factors. Lung Cancer 2013;82:95-102.

61. Eichhorn F, Winter H. How to handle oligometastatic disease in nonsmall cell lung cancer. Eur Respir Rev 2021;30:200234.

62. Londero F, Grossi W, Morelli A, et al. Surgery versus stereotactic radiotherapy for treatment of pulmonary metastases. A systematic review of literature. Future Sci OA 2020;6:FSO471.

63. Kessel KA, Grosser RCE, Kraus KM, et al. Stereotactic body radiotherapy (SBRT) in patients with lung metastases - prognostic factors and long-term survival using patient self-reported outcome (PRO). BMC Cancer 2020;20:442.

64. De Rose F, Cozzi L, Navarria P, et al. Clinical Outcome of Stereotactic Ablative Body Radiotherapy for Lung Metastatic Lesions in Non-small Cell Lung Cancer Oligometastatic Patients. Clin Oncol (R Coll Radiol) 2016;28:13-20.

65. Siva S, Slotman BJ. Stereotactic Ablative Body Radiotherapy for Lung Metastases: Where is the Evidence and What are We Doing With It? Semin Radiat Oncol 2017;27:229-39.

66. Lee J, Shin IS, Yoon WS, et al. Comparisons between radiofrequency ablation and stereotactic body radiotherapy for liver malignancies: Meta-analyses and a systematic review. Radiother Oncol 2020;145:63-70.
67. Gray PJ, Mak RH, Yeap BY, et al. Aggressive therapy for patients with non-small cell lung carcinoma and synchronous brain-only oligometastatic disease is associated with long-term survival. Lung Cancer 2014;85:239-44.

68. Flannery TW, Suntharalingam M, Regine WF, et al. Longterm survival in patients with synchronous, solitary brain metastasis from non-small-cell lung cancer treated with radiosurgery. Int J Radiat Oncol Biol Phys 2008;72:19-23.

69. Hu C, Chang EL, Hassenbusch SJ 3rd, et al. Nonsmall cell lung cancer presenting with synchronous solitary brain metastasis. Cancer 2006;106:1998-2004.

70. Yang SY, Kim DG, Lee SH, et al. Pulmonary resection in patients with nonsmall-cell lung cancer treated with gamma-knife radiosurgery for synchronous brain metastases. Cancer 2008;112:1780-6.

71. Nikitas J, Roach M, Robinson C, et al. Treatment of oligometastatic lung cancer with brain metastases using stereotactic radiosurgery (SRS) and stereotactic body radiation therapy (SBRT). Clin Transl Radiat Oncol 2020;21:32-5.

72. Jackson WC, Tao Y, Mendiratta-Lala M, et al. Comparison of Stereotactic Body Radiation Therapy and Radiofrequency Ablation in the Treatment of Intrahepatic Metastases. Int J Radiat Oncol Biol Phys 2018;100:950-8.

73. Petrella F, Spaggiari L. Comparison of pulmonary metastasectomy and stereotactic body radiation therapy for the treatment of lung metastases. J Thorac Dis 2019;11:S280-2.

74. Soni PD, Hartman HE, Dess RT, et al. Comparison of Population-Based Observational Studies With Randomized Trials in Oncology. J Clin Oncol 2019;37:1209-16.

75. Hendriks LEL, Dooms C, Berghmans T, et al. Defining oligometastatic non-small cell lung cancer: A simulated multidisciplinary expert opinion. Eur J Cancer 2019;123:28-35.

76. Hong JC, Ayala-Peacock DN, Lee J, et al. Classification for long-term survival in oligometastatic patients treated with ablative radiotherapy: A multi-institutional pooled analysis. PLoS One 2018;13:e0195149.

77. Fode MM, Høyer M. Survival and prognostic factors in 321 patients treated with stereotactic body radiotherapy for oligo-metastases. Radiother Oncol 2015;114:155-60.

78. Tanadini-Lang S, Rieber J, Filippi AR, et al. Nomogram based overall survival prediction in stereotactic body radiotherapy for oligo-metastatic lung disease. Radiother Oncol 2017;123:182-8.

79. Ashworth AB, Senan S, Palma DA, et al. An individual 
patient data metaanalysis of outcomes and prognostic factors after treatment of oligometastatic non-small-cell lung cancer. Clin Lung Cancer 2014;15:346-55.

80. Voltolini L, Rapicetta C, Luzzi L, et al. Surgical treatment of synchronous multiple lung cancer located in a different lobe or lung: high survival in node-negative subgroup. Eur J Cardiothorac Surg 2010;37:1198-204.

81. Dingemans AC, Hendriks LEL, Berghmans T, et al. Definition of Synchronous Oligometastatic Non-Small Cell Lung Cancer-A Consensus Report. J Thorac Oncol 2019;14:2109-19.

82. Sundahl N, Gillessen S, Sweeney C, et al. When What You See Is Not Always What You Get: Raising the Bar of Evidence for New Diagnostic Imaging Modalities. Eur Urol 2021;79:565-7.

83. Levy A, Hendriks LEL, Berghmans T, et al. EORTC Lung Cancer Group survey on the definition of NSCLC synchronous oligometastatic disease. Eur J Cancer 2019;122:109-14.

84. Berghmans T, Lievens Y, Aapro M, et al. European Cancer Organisation Essential Requirements for Quality Cancer Care (ERQCC): Lung cancer. Lung Cancer 2020;150:221-39.

85. Theelen WSME, Peulen HMU, Lalezari F, et al. Effect of Pembrolizumab After Stereotactic Body Radiotherapy vs Pembrolizumab Alone on Tumor Response in Patients With Advanced Non-Small Cell Lung Cancer: Results of the PEMBRO-RT Phase 2 Randomized Clinical Trial. JAMA Oncol 2019;5:1276-82.

86. Sundahl N, De Wolf K, Kruse V, et al. Phase 1 Dose Escalation Trial of Ipilimumab and Stereotactic Body Radiation Therapy in Metastatic Melanoma. Int J Radiat Oncol Biol Phys 2018;100:906-15.

87. Sundahl N, Seremet T, Van Dorpe J, et al. Phase 2 Trial of Nivolumab Combined With Stereotactic Body Radiation Therapy in Patients With Metastatic or Locally Advanced
Inoperable Melanoma. Int J Radiat Oncol Biol Phys 2019;104:828-35.

88. Sundahl N, Vandekerkhove G, Decaestecker K, et al. Randomized Phase 1 Trial of Pembrolizumab with Sequential Versus Concomitant Stereotactic Body Radiotherapy in Metastatic Urothelial Carcinoma. Eur Urol 2019;75:707-11.

89. Herrera FG, Bourhis J, Coukos G. Radiotherapy combination opportunities leveraging immunity for the next oncology practice. CA Cancer J Clin 2017;67:65-85.

90. Vanpouille-Box C, Alard A, Aryankalayil MJ, et al. DNA exonuclease Trex1 regulates radiotherapy-induced tumour immunogenicity. Nat Commun 2017;8:15618.

91. Powles T, Durán I, van der Heijden MS, et al. Atezolizumab versus chemotherapy in patients with platinum-treated locally advanced or metastatic urothelial carcinoma (IMvigor211): a multicentre, open-label, phase 3 randomised controlled trial. Lancet 2018;391:748-57.

92. Gandhi L, Rodríguez-Abreu D, Gadgeel S, et al. Pembrolizumab plus Chemotherapy in Metastatic NonSmall-Cell Lung Cancer. N Engl J Med 2018;378:2078-92.

93. Lieverse RIY, Van Limbergen EJ, Oberije CJG, et al. Stereotactic ablative body radiotherapy (SABR) combined with immunotherapy (L19-IL2) versus standard of care in stage IV NSCLC patients, ImmunoSABR: a multicentre, randomised controlled open-label phase II trial. BMC Cancer 2020;20:557.

94. Dingemans AC, Hendriks LEL, Berghmans T, et al. Definition of Synchronous Oligometastatic Non-Small Cell Lung Cancer-A Consensus Report. J Thorac Oncol 2019;14:2109-19.

95. Nevens D, Kindts I, Defourny N, et al. The financial impact of SBRT for oligometastatic disease: A population-level analysis in Belgium. Radiother Oncol 2020;145:215-22.
Cite this article as: Sundahl N, Lievens Y. Radiotherapy for oligometastatic non-small cell lung cancer: a narrative review. Transl Lung Cancer Res 2021;10(7):3420-3431. doi: 10.21037/ tlcr-20-1051 